\title{
Hepatic safety of ketoconazole in Cushing's syndrome: results of a Compassionate Use Programme in France
}

Jacques Young 1,2,3, Jérôme Bertherat ${ }^{4}$, Marie Christine Vantyghem ${ }^{5}$, Olivier Chabre ${ }^{6,7,8}$, Salima Senoussi ${ }^{9}$, Rita Chadarevian ${ }^{9,10}$ and Frédéric Castinetti ${ }^{11}$ on behalf of the Compassionalte use Programme*

${ }^{1}$ Univ Paris-Sud, Faculté de Médecine Paris-Sud, Le Kremlin Bicetre, France, ${ }^{2}$ Service d'Endocrinologie et des Maladies de la Reproduction, Assistance Publique-Hôpitaux de Paris, Hôpital Bicêtre, Le Kremlin Bicetre, France, ${ }^{3}$ INSERM U1185, Le Kremlin-Bicetre, France, ${ }^{4}$ Department of Endocrinology, Metabolism, and Diabetes, Institut National de la Santé et de la Recherche Médicale (INSERM) Unit 1016, Institut Cochin, Centre National de la Recherche Scientifique (CNRS) UMR 8104, Paris, France, ${ }^{5}$ Service d'Endocrinologie et Maladies Métaboliques, Hôpital Huriez, Centre Hospitalier Régional Universitaire de Lille, INSERM U1190, EGID, Lille, France, ${ }^{6}$ Service d'Endocrinologie-Diabétologie-Nutrition, CHU Grenoble-Alpes, Grenoble, France, ${ }^{7}$ Faculté de Médecine Université Grenoble-Alpes, Grenoble, France, ${ }^{8}$ INSERM U 1036, iRTSV-BCI, CEA-Grenoble, Grenoble, France, ${ }^{9} \mathrm{HRA}$ France, Paris, France, ${ }^{10}$ Service d'endocrinologiemétabolisme, Assistance Publique-Hôpitaux de Paris, Hôpital Pitié-Salpêtrière, Paris, France, and ${ }^{11}$ Department of Endocrinology, Aix Marseille Universite, CNRS UMR7286, Assistance Publique-Hopitaux de Marseille, La Conception Hospital, Marseille, France

*(Details of the Compassionate Use Programme participants is presented in the Acknowledgements section)

Correspondence should be addressed to J Young

Email jacques.young@aphp.fr

\begin{abstract}
Objective: Ketoconazole (KTZ) is one of few available treatments for Cushing's syndrome (CS). Although KTZ has been associated with severe hepatotoxicity, little information is available about hepatic safety in CS. The aim of this study was to document changes in liver function in patients with CS treated with KTZ.

Design: An observational prospective French cohort study (Compassionate Use Programme (CUP)).

Methods: Enrolled patients were stratified into a KTZ-naive cohort and a cohort already treated by another formulation of ketoconazole (KTZ-switch cohort). Liver function markers (alanine transaminase (ALT), aspartate transaminase (AST), alkaline phosphatase, $\gamma$-glutamyltransferase and bilirubin) were monitored at regular intervals. Patients with ALT $>3 \times$ ULN (upper limit of normal), total bilirubin $>2 \times U L N$ or both ALP $>2 \times U L N$ and ALT $>U L N$ were considered to have liver injury. Results: Overall, 108 patients were analysed (47 KTZ-naïve; 61 KTZ-switch). The median KTZ dose was $600 \mathrm{mg} /$ day. Most abnormalities observed were asymptomatic mild increases of liver enzymes. Four patients in the KTZ-naïve cohort (8.5\%) and two in the KTZ-switch cohort (3.3\%) developed liver injury, considered related to KTZ in three cases (all KTZ-naïve in the first month of treatment). Five patients had mild liver function abnormalities at baseline and two had proven liver metastases. Two patients recovered on discontinuation of KTZ and the remaining patient died of unrelated causes. Conclusions: These findings highlight the need for close monitoring of liver enzymes especially during the first six months of treatment. Liver enzyme abnormalities usually occurred within four weeks were asymptomatic and could
\end{abstract} be reversed on timely discontinuation of KTZ.

\section{Introduction}

Endogenous Cushing's syndrome (CS) is an endocrine disorder caused by prolonged exposure to corticosteroids produced by the adrenal cortex $(1,2)$. The first-line

www.eje-online.org

https://doi.org/10.1530/EJE-17-0886
C) 2018 European Society of Endocrinology Printed in Great Britain treatment of CS is removal of the pituitary adenoma, ectopic neuroendocrine-secreting ACTH tumour or adrenocortical tumour $(1,3)$. Medical treatment may be 
provided if needed before surgery to prepare the patient or with radiotherapy to cover the period before it is effective (3). Medical treatment is also used when surgery is not possible, in case of surgery failure or of recurrence of CS after surgery. In this context, drugs that inhibit cortisol synthesis may be of use (4). One such drug is ketoconazole (KTZ), a drug originally developed and marketed as an antifungal agent (5), which can inhibit several of the cytochrome P450 enzymes that play a central role in corticosteroid synthesis and metabolism $(6,7,8,9,10)$. This agent has been used for decades in the treatment of CS on an off-label basis. Although KTZ is effective in lowering circulating cortisol and in reducing the clinical manifestations of CS, its use is challenging due to the risk of severe hepatotoxicity $(11,12)$, a rare but potentially life-threatening complication.

Although moderate and asymptomatic increases in liver enzymes are common in patients treated with KTZ ( $>1$ case/10 treated subjects), severe hepatotoxicity is in fact rare $(13,14,15)$. This has principally been reported when used as antifungal therapy in community medicine in patients whose liver function was not monitored adequately as specified in the prescribing information $(13,16,17)$. In particular, in cases which were fatal, the drug had been continued after the onset of jaundice and other clinical symptoms of hepatitis and liver failure $(13,17)$. Given the hepatotoxic risk associated with KTZ, and the availability of alternative antifungal agents with equivalent antifungal efficacy and lower toxicity, the European Medicines Agency (EMA) withdrew marketing authorisation for KTZ as an antifungal drug in 2013 (18). This left patients with CS successfully managed with KTZ without medical treatment, especially in France, where the marketing authorisation for the oral formulations of KTZ (Nizoral; Janssen Cilag) as an antifungal drug was suspended in 2011. However, in the absence of therapeutic alternatives for patients with CS, oral KTZ remained available exclusively for these patients on a compassionate use basis while stocks lasted until June 2014. At the same time, a new manufacturer (HRA Pharma) filed a new drug application in Europe for the use of KTZ specifically for the treatment of CS in adults and adolescents over twelve years of age. While awaiting marketing authorisation in Europe and the launch of KTZ HRA in France in March 2015, a formal compassionate use programme (CUP) was established in France, which ran from June 2014 to March 2015. As a condition of the implementation of the CUP, the French health authorities requested that the safety of KTZ in the treatment of CS be monitored throughout the CUP. In contrast to randomised Phase III clinical trials, the temporary authorisation of use in the context of a CUP is not intended to provide definitive information on the efficacy of the drug in question, but to document tolerability through an observational study of a cohort of patients followed in a real-world setting of everyday care.

The objective of the present study is to document the hepatic safety of KTZ in a cohort of patients with CS receiving KTZ in a real-life treatment setting and to compare liver safety according to the treatment duration.

\section{Subjects and methods}

This was a prospective, longitudinal, observational study of the French CUP cohort treated in a real-world setting with KTZ. Patients were enrolled between June 2014 and February 2015 and the database locked on 9th April 2015.

The study was conducted in conformity with the Guidelines for Good Clinical Practice and the principles laid down in the Declaration of Helsinki (1964) including all subsequent amendments and all relevant national legislation and guidelines for compassionate use. The study protocol was submitted and approved by the French health authorities as part of the marketing application for KTZ from HRA Pharma. All patient data in the study database were rendered anonymous. Written informed consent was obtained from each patient prior to inclusion.

\section{Participants}

All hospital endocrinologists wishing to prescribe KTZ could participate in the CUP by filing a request to HRA Pharma for an individual patient with CS. According to the CUP, physicians were expected to provide information regarding the follow-up of all included patients. However, even if they failed to do so, HRA Pharma continued to deliver the drug in order to ensure continuity of treatment for the patient.

Patients with a diagnosis of CS from any cause and for whom baseline information on disease history was available were eligible for inclusion in the CUP. It was verified that all eligible patients had no contraindication to KTZ and were not taking any contraindicated associated medication, as specified in the prescribing information. In particular, patients with acute or chronic liver disease or with serum liver function markers above two times the upper limit of normal (ULN) were excluded. Before enrolment, all eligible patients were provided with information on the goals of the study and on follow-up procedures, and were invited to provide written informed 
consent. Enrolled patients were prospectively stratified into two cohorts, one who had already been treated by the previously marketed product (KTZ-switch cohort) for $\geq 1$ month of inclusion and a second cohort of patients who had not received treatment with $\mathrm{KTZ}<1$ month before inclusion (KTZ-naïve cohort). In the KTZ-naïve cohort, patients could be included if they had started treatment up to four weeks prior to the inclusion visit. The purpose of this stratification was to take into account the impact of overall treatment duration on liver safety and the recommended monitoring schedule. In addition, for the purposes of certain analyses, the switch cohort was subdivided into two subgroups, one previously treated for over six months and the other previously treated from between one and six months.

\section{Treatments}

All patients received KTZ $200 \mathrm{mg}$ tablets (HRA Pharma) administered according to the prescribing information. For patients in the KTZ-naïve cohort, the proposed initial dose was $400-600 \mathrm{mg} /$ day in two or three divided doses, which could then be adjusted at the physician's discretion as a function of the change in cortisol levels (determined from 24-h urinary free cortisol levels assayed every few days) and of the tolerability of the treatment. If necessary, the dose could be up-titrated to $800-1200 \mathrm{mg} /$ day in two or three divided doses. All changes in treatment occurring over the study period had to be documented, including changes to KTZ dose, changes in adjunctive treatments and discontinuation of KTZ. Patients in the KTZ-switch cohort continued at their previous KTZ dose and subsequent dose change could be performed as described earlier.

\section{Study procedures}

As this was an observational study under usual care conditions, there were no formal or mandatory study procedures. However, some guidance was provided on the conduct of the study. The study involved an inclusion visit and several follow-up visits for monitoring of serum liver function markers. At the inclusion visit, the physician informed the patient about the risks of the treatment in general and about signs of hepatotoxicity in particular and obtained written consent to participate. A blood sample was taken for assay of liver function markers. In the absence of liver function abnormalities or contraindications specified in the prescribing information, treatment with KTZ from HRA Pharma was started.
With respect to follow-up visits, patients in the KTZnaïve cohort were to be monitored every week for the first month and once a month thereafter for six months. For patients in the KTZ-switch cohort, the first visit was planned one month after the switch if the patient had been treated with KTZ between one and six months, and three months after the switch if the patient had been treated for over six months. After six months, visits were planned every three months.

\section{Outcome variables}

The evolution of serum markers of liver function was one of the primary outcomes of this observational study. These were to be measured at inclusion and at each follow-up visit at the physicians' discretion. The markers evaluated were alanine aminotransferase (ALT), aspartate aminotransferase (AST), $\gamma$-glutamyltransferase (GGT), alkaline phosphatase (ALP) and total bilirubin (TB). Assays were performed in local accredited laboratories, which applied their own definitions of the normal range. Levels of each serum marker were expressed as multiples of the upper limit of the normal range (ULN). If elevations were observed, the ratio of ALT to ALP (both expressed as multiples of the ULN) was calculated. In the absence of a measurement of ALT, AST was used instead. This ratio (R) was used to define the pattern of liver injury.

The patient was considered to have liver injury if one of the four following conditions was met: (i) ALT $>3 \times \mathrm{ULN}$, (ii) AST $>3 \times \mathrm{ULN}$ if $\mathrm{ALT} \leq 3 \times \mathrm{ULN}$, (iii) total bilirubin $>2 \times$ ULN or (iv) both ALP $>2 \times$ ULN and ALT $>$ ULN. The pattern of injury was considered to be hepatocellular if ALT $>3 \times$ ULN and ALP was normal or, if ALT and ALP were both increased and $R$ was $>5$. The pattern was considered to be cholestatic if ALP $>2 \times$ ULN and ALT was normal or, if ALT and ALP were both increased and $R$ was $\leq 2$. If both ALP and transaminases were elevated and $R$ was $>2$ but $\leq 5$, the pattern was considered to be mixed. Patients who had abnormal liver tests values but which remained below these thresholds were defined as just having liver function test abnormalities. All cases were reviewed by an expert hepatologist who assessed a possible causal relationship to KTZ treatment.

If the level of any liver function marker was $\geq 3 \times \mathrm{ULN}$, KTZ had to be discontinued immediately and irrevocably. If the levels were $>\mathrm{ULN}$ but $<3 \times \mathrm{ULN}$, the dose of KTZ had to be decreased by $\geq 200 \mathrm{mg}$ per day and the marker monitored tightly until the liver function test normalised and treatment continued thereafter at the lower dose. 
Investigators were asked to record any adverse drug reaction occurring under KTZ treatment on the case report form (CRF). The investigators' verbatim was consolidated and classified using standardised MedRA terminology.

\section{Data quality}

Physicians were expected to record all data relating to the study in a paper CRF, but this was not monitored and, in practice, the CRF was not filled in systematically. Although there was no formal monitoring, on-site visits were organised for six sites who included more than three patients to help CRF collection, and telephone contact was made with a further eight sites including three or four patients in order to facilitate retrieval of CRFs.

\section{Statistical analysis}

The analysis was performed on the all-treated set, defined as all patients participating in the CUP, treated with KTZ and for whom at least one post-treatment measure of liver function markers was available. Analyses were performed separately for the KTZ-naïve cohort and the KTZ-switch cohort and for both cohorts combined. The analysis was essentially descriptive. Continuous variables are presented as mean values \pm standard deviations (or with $95 \%$ confidence intervals) or as median values with range. Categorical variables are presented as frequency counts and percentages. Statistical programming and analyses were performed using SAS, version 9.4.

\section{Results}

\section{Participants}

A total of 212 treatment requests were received from 133 endocrinologists in 100 hospitals. In 191 cases (90.1\%), the patient fulfilled the eligibility criteria and was enrolled (Fig. 1). The remaining 21 patients were not enrolled, due to abnormalities in liver function tests at screening (12 patients) or requests for use in indications other than CS (such as prostatic carcinoma or hypercalcaemiahypercalciuria; 9 patients). Of these patients, 108 (56.5\%) were treated and at least one post-treatment measure of liver function markers was provided by the treating physician. These patients (75\% women, mean age 51.3 (range: 11-86)) constitute the all-treated set. Forty-seven patients in the all-treated sets were KTZ-naïve and the remaining 61 had previously been treated with KTZ. Of these 61 previously treated patients, 51 had been treated for over six months.
The mean duration of KTZ treatment before inclusion in the CUP was $50.7 \pm 62.0$ months (median: 26 months; range: 2-286 months). Patients in the switch group had been diagnosed for longer (median: 69.7 months vs 2.5 months). The main reasons for using ketoconazole are presented in Table 1. At the time of inclusion, 28 patients were receiving another cortisone-lowering drug, namely twenty patients treated with metyrapone, seven with mitotane and five with cabergoline (some patients received more than one such drug).

In total, 39 patients in the all-treated set (36.1\%) discontinued the CUP. These included nine post-treatment deaths, none of which were considered to be related to KTZ treatment, seven discontinuations for adverse events considered to be possibly related to KTZ, seven patients who were scheduled for surgery and four patients for whom KTZ treatment was ineffective. For the remaining twelve patients, the reasons for discontinuation were not related to KTZ. The adverse events leading to treatment discontinuation included three cases of liver injury (discussed below), two cases of adrenal insufficiency and one case each of alopecia and generalised pruritus.

\section{Ketoconazole treatment}

The mean dose of KTZ and the mean treatment duration for the all-treated set are presented in Table 2. Most patients required upward titration of the initial dose, and the median final dose was close to $600 \mathrm{mg}$ in both the KTZnaïve and the KTZ-switch cohorts (range 200-1200 mg).

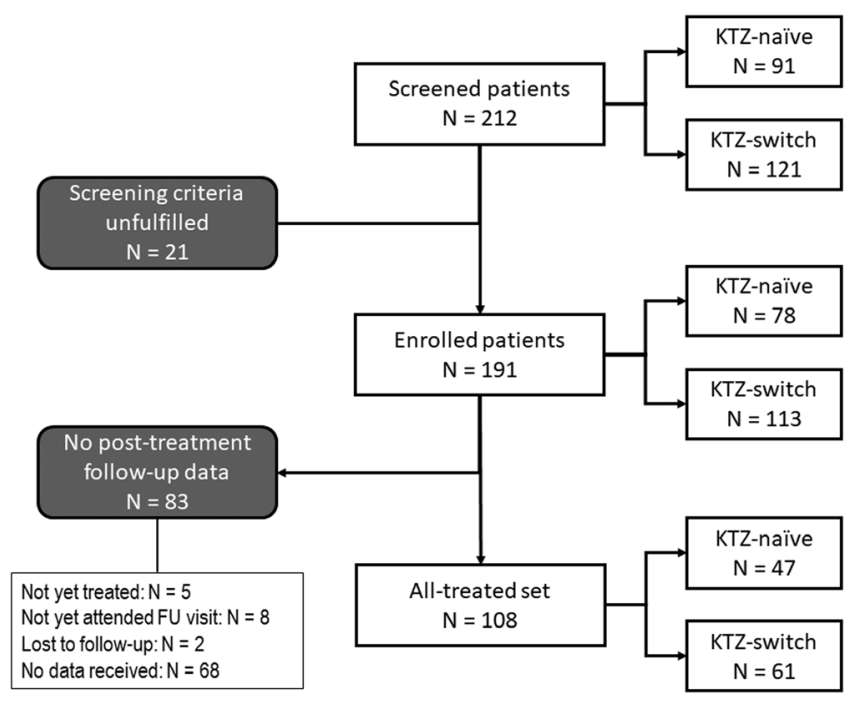

Figure 1

Patient distribution. FU, follow-up; KTZ, ketoconazole. 
Table 1 Causes of CS and medical indication of ketoconazole prescription in the all-treated set. Percentages are calculated with respect to the number of documented cases (46 in the KTZ-naïve cohort and 58 in the KTZ-switch cohort); the reason for treatment was not documented for the four remaining cases. The 'other' category may include, for example, patient refusal for surgery.

\begin{tabular}{|c|c|c|c|c|c|}
\hline & Awaiting surgery & Surgery ineffective & $\begin{array}{l}\text { Not eligible for } \\
\text { surgery }\end{array}$ & Other & Total \\
\hline \multicolumn{6}{|l|}{ KTZ-naïve cohort $(n=47)$} \\
\hline Total & $13(28.3 \%)$ & $7(15.2 \%)$ & $12(26.1 \%)$ & $14(30.4 \%)$ & $46(100 \%)$ \\
\hline $\mathrm{ACC}$ & $2(4.3 \%)$ & None & $4(8.7 \%)$ & $2(4.3 \%)$ & $8(17.4 \%)$ \\
\hline Cushing's disease & $8(17.4 \%)$ & $7(15.2 \%)$ & $7(15.2 \%)$ & $1(2.2 \%)$ & $23(50.0 \%)$ \\
\hline Ectopic ACTH secretion & $2(4.3 \%)$ & None & None & $10(21.7 \%)$ & $12(41.7 \%)$ \\
\hline ACTH-independent CS & None & None & $1(2.2 \%)$ & None & $1(2.2 \%)$ \\
\hline Other & $1(2.2 \%)$ & None & None & $1(2.2 \%)$ & $2(4.3 \%)$ \\
\hline \multicolumn{6}{|l|}{ KTZ-switch cohort $(n=61)$} \\
\hline Total & $4(6.9 \%)$ & $24(41.4 \%)$ & $22(37.9 \%)$ & $8(13.8 \%)$ & $58(100 \%)$ \\
\hline $\mathrm{ACC}$ & None & None & None & None & None \\
\hline Cushing's disease & $4(6.9 \%)$ & $24(41.4 \%)$ & $14(24.1 \%)$ & $5(8.6 \%)$ & $47(81.0 \%)$ \\
\hline Ectopic ACTH secretion & None & None & $3(5.2 \%)$ & $2(3.4 \%)$ & $5(8.6 \%)$ \\
\hline ACTH-independent CS & None & None & $5(8.6 \%)$ & $1(1.7 \%)$ & $6(10.3 \%)$ \\
\hline Other & None & None & None & None & None \\
\hline
\end{tabular}

Percentages are calculated with respect to the number of documented cases (46 in the KTZ-naïve cohort and 58 in the KTZ-switch cohort); the reason for treatment was not documented for the four remaining cases. The 'other' category may include, for example, patient refusal for surgery.

ACC, adrenal cell carcinoma; ACTH, adrenocorticotropic hormone; CS, Cushing's syndrome.

\section{Liver function tests}

The evolution of liver enzymes from baseline to the last available visit for three groups (KTZ-naïve, KTZ-switch with a previous duration of treatment between 1 and 6 months and KTZ-switch with a previous duration of treatment $\geq 6$ months) is presented in Table 3. Elevations of all the markers studied tended to be more frequent and greater in the KTZ-naïve cohort than in the KTZ-switch cohort. Elevations were most commonly observed for GGT, whereas elevations in TB were only observed in one patient. The highest elevation of ALT observed was $7 \times$ ULN measured one week after inclusion and concerned a patient in the KTZ-naïve group with an ectopic ACTH syndrome and liver metastases (Patient 3; Table 4), and who also presented elevations of ALT and GGT at baseline. In this patient, on-treatment elevations were also observed for AST, ALP and GGT. Overall, around 10\% of patients presented elevations of AST or ALT at baseline, although these did not exceed $2 \times \mathrm{ULN}$, consistent with the eligibility criteria for the study.

Figure 2 presents the time-course of the changes in ALT, AST and ALP in individual patients. In the KTZ-naïve cohort, the majority of elevations >ULN corresponded to sharp rises in ALT levels occurring within the first month of treatment. In contrast, in the KTZ-switch cohort who, by definition, have been exposed to a longer treatment duration, such abrupt changes were not observed, and the elevations above the ULN generally corresponded to a modest rise from a level already close to the threshold and at a similar rate throughout the study period. In other patients in this cohort, modest falls in ALT levels of a similar magnitude to underneath the threshold were observed. Very similar patterns were observed for changes in AST and ALP, although elevations were generally of lower magnitude (Fig. 2).

\section{Liver injury}

Six patients, four in the KTZ-naïve cohort (8.5\%) and two in the KTZ-switch cohort (3.3\%), fulfilled the prespecified criteria for liver injury (Table 4). In all four cases in the

Table 2 Treatment with ketoconazole during the CUP (all-treated set, see also Fig. 1).

\begin{tabular}{|c|c|c|}
\hline & KTZ naïve $(n=47)$ & KTZ switch $(n=61)$ \\
\hline \multicolumn{3}{|c|}{ Final dose of KTZ (mg/day) } \\
\hline Mean \pm S.D. & $679 \pm 277$ & $629 \pm 265$ \\
\hline Median (range) & $600(200-1200)$ & $600(200-1200)$ \\
\hline \multicolumn{3}{|c|}{ Treatment duration in CUP (months) ${ }^{\mathrm{a}}$} \\
\hline$n$ & 46 & 61 \\
\hline$<1$ month & $15(32.6 \%)$ & $3(4.9 \%)$ \\
\hline$\geq 1$ to $<6$ months & $27(58.7 \%)$ & $18(29.5 \%)$ \\
\hline$\geq 6$ months & $4(8.7 \%)$ & $40(65.6 \%)$ \\
\hline
\end{tabular}

${ }^{a}$ The duration of exposure to KTZ is calculated as the difference between the KTZ start date in the CUP and the last KTZ dosing date (or the last date of data entry in the database if the last dose date was not available).

The $n$ values at the top of each box correspond to the number of patients for whom data were available. 
Table 3 Evolution of liver function markers over the course of the study. The denominator ' $n$ ' indicates the number of patients with a baseline and at least one follow-up determination of the marker in question. Data present the numbers of patients reaching the indicated level at any stage over the study.

\begin{tabular}{|c|c|c|c|}
\hline & KTZ-naïve $(n=47)$ & KTZ-switch (1-6 months) $(n=10)$ & KTZ-switch (>6 months) $(n=51)$ \\
\hline ALT & $n=31$ & $n=4$ & $n=35$ \\
\hline Abnormal value at baseline & 6 & None & 5 \\
\hline$>$ ULN at least once post-baseline & $10(32.3 \%)$ & None & $8(22.9 \%)$ \\
\hline $\mathrm{ULN}<\mathrm{ALT}<2 \times \mathrm{ULN}$ & 3 & 0 & 7 \\
\hline $2 \times U L N \leq A L T<3 \times U L N$ & 3 & 0 & 1 \\
\hline $3 \times \mathrm{ULN} \leq \mathrm{ALT}<5 \times \mathrm{ULN}$ & 0 & 0 & 0 \\
\hline $\mathrm{ALT} \geq 5 \times \mathrm{ULN}$ & 4 & 0 & 0 \\
\hline AST & $n=31$ & $n=4$ & $n=36$ \\
\hline Abnormal value at baseline & 4 & None & 2 \\
\hline$>$ ULN at least once post-baseline & $12(38.7 \%)$ & None & $4(11.1 \%)$ \\
\hline $\mathrm{N}<\mathrm{AST}<2 \times \mathrm{ULN}$ & 9 & 0 & 4 \\
\hline $2 \times \mathrm{ULN} \leq \mathrm{AST}<3 \times \mathrm{ULN}$ & 2 & 0 & 0 \\
\hline $3 \times U L N \leq A S T<5 \times U L N$ & 1 & 0 & 0 \\
\hline ALP & $n=29$ & $n=4$ & $n=30$ \\
\hline Abnormal value at baseline & 5 & 1 & 1 \\
\hline >ULN at least once post-baseline & $10(34.5 \%)$ & $1(25.0 \%)$ & $4(13.3 \%)$ \\
\hline ULN $<$ ALP $<2 \times$ ULN & 6 & 1 & 4 \\
\hline $2 \times U L N \leq A L P<3 \times U L N$ & 3 & 0 & 0 \\
\hline $3 \times U L N \leq A L P<5 \times U L N$ & 1 & 0 & 0 \\
\hline GGT & $n=30$ & $n=5$ & $n=32$ \\
\hline Abnormal value at baseline & 12 & 1 & 6 \\
\hline$>$ ULN at least once post-baseline & $18(60.0 \%)$ & $1(20.0 \%)$ & $10(31.2 \%)$ \\
\hline $\mathrm{ULN}<\mathrm{GGT}<2 \times \mathrm{ULN}$ & 7 & 0 & 6 \\
\hline $2 \times \mathrm{ULN} \leq \mathrm{GGT}<3 \times \mathrm{ULN}$ & 3 & 0 & 1 \\
\hline $3 \times \mathrm{ULN} \leq \mathrm{GGT}<5 \times \mathrm{ULN}$ & 3 & 0 & 2 \\
\hline $\mathrm{GGT} \geq 5 \times \mathrm{ULN}$ & 5 & 1 & 1 \\
\hline Total bilirubin & $n=20$ & $n=1$ & $n=24$ \\
\hline Abnormal value at baseline & None & None & None \\
\hline$>$ ULN at least once post-baseline & $1(0.5 \%)$ & None & None \\
\hline $3 \times \mathrm{ULN} \leq \mathrm{GGT}<5 \times \mathrm{ULN}$ & 1 & 0 & 0 \\
\hline
\end{tabular}

The denominator ' $n$ ' indicates the number of patients with a baseline and at least one follow-up determination of the marker in question. Data present the numbers of patients reaching the indicated level at any stage over the study.

ALP, alkaline phosphatase; ALT, alanine aminotransferase; AST, aspartate aminotransferase; GGT, gamma-glutamyl-transferase.

KTZ-naïve cohort, liver injury occurred during the first month of treatment. One patient in the KTZ-naïve cohort (Patient 2) presented a hepatocellular pattern of injury (ALT $\geq 3 \times U L N$ ) associated with non-specific clinical signs (nausea and vomiting), within one month of starting KTZ. Jaundice did not develop. Upon discontinuation of KTZ, these symptoms disappeared and transaminases normalised. The other cases in the KTZ-naïve cohort were asymptomatic and showed mixed patterns of liver injury. All had mild abnormalities in liver function tests at baseline, together with documented metastases in two cases. The two cases in the KTZ-switch cohort both presented a cholestatic pattern of injury and abnormalities in liver function tests at baseline. With respect to dose, all patients who developed liver injury in the naïve group and one of the two patients in the switch group had been exposed to a dose of $\geq 800 \mathrm{mg}$, either as the initial dose or as a rapid up-titration, whereas this was the case for less than a third of patient without liver injury. Information on the distribution of liver injury and elevations of transaminases with respect to maximal KTZ dose is provided in the Supplementary data online (see section on Supplementary data given at the end of this article).

One of these two cases of liver injury (Patient 5), with a bilirubin level close to the ULN at inclusion subsequently developed jaundice. In three cases (Patients 1, 2 and 3), all in the KTZ-naïve group) KTZ was discontinued due to a likely causal relationship with treatment. In the remaining patient in the KTZ-naïve group, who was terminally ill, the dose was increased since hepatic dysfunction was considered to be less detrimental to the patient's overall well-being than uncontrolled CS. In the two cases in the KTZ-switch group with cholestatic liver injury, the dose was continued. Following a further rise in liver enzymes, the dose of KTZ was subsequently reduced to $\leq 600 \mathrm{mg} /$ day. 


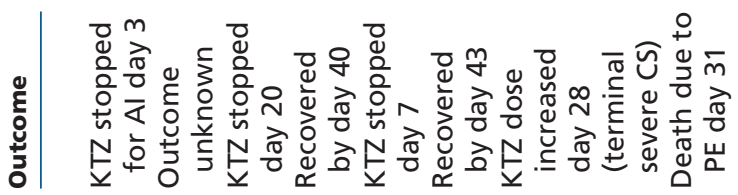

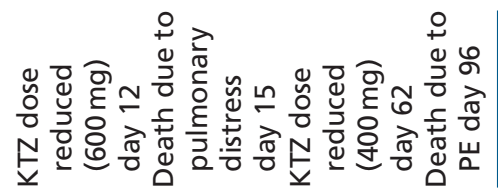

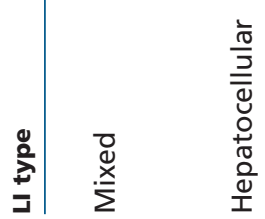

$\begin{array}{ll}\bar{d} & \bar{d} \\ \dot{\mathbb{d}} & \dot{x} \\ \dot{x} & \dot{x}\end{array}$

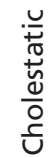

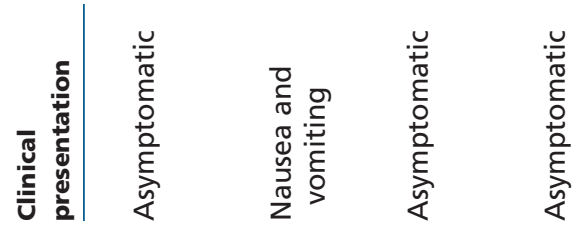

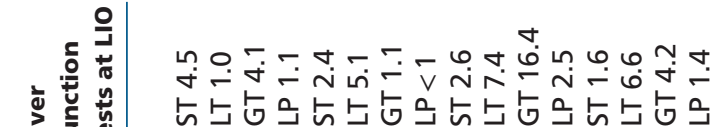

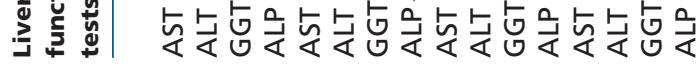

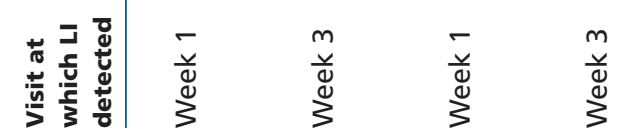

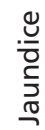

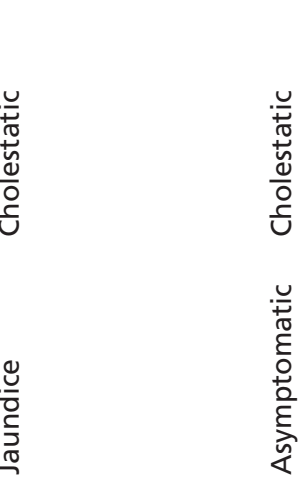

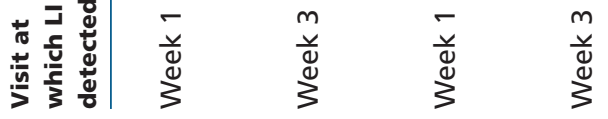

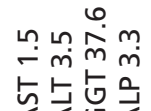

을

iा

인

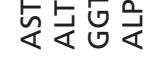

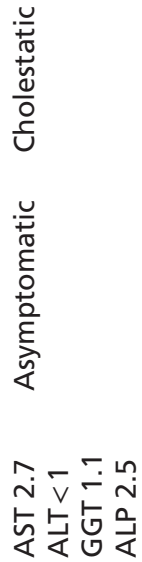

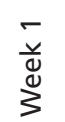

이

$\stackrel{\stackrel{8}{ }}{1}$

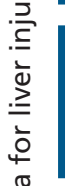

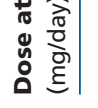

요윰

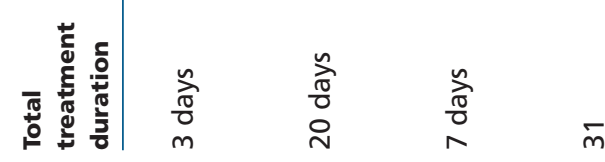

$\underset{\frac{n}{\sigma}}{0}$
$\stackrel{n}{\circ}$

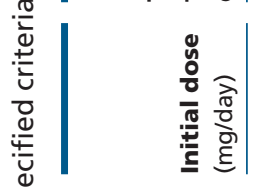

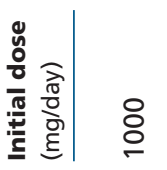

용 웅

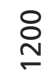

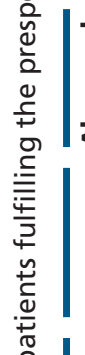

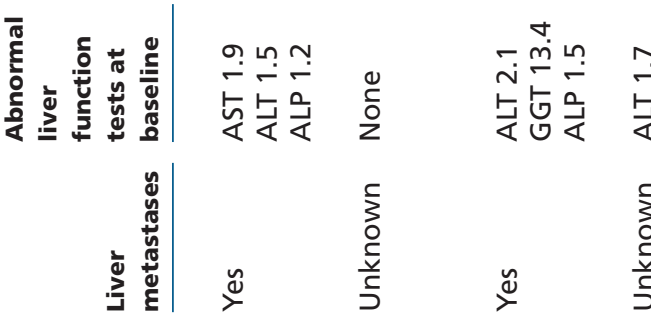

눌웜

它

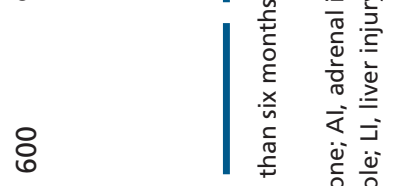

¿্য

¿

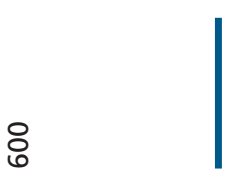

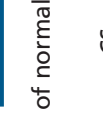

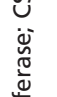

年

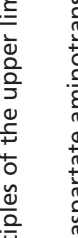

$\stackrel{\text { I }}{\vec{z}}$

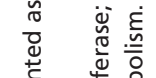

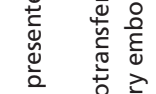

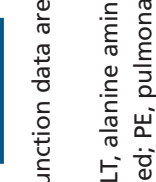

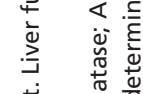

응 ํํㅇํำ

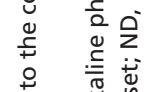

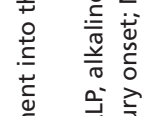

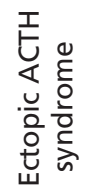

$\begin{array}{ll}\frac{5}{5} & \frac{0}{0} \\ 0 & \frac{0}{0}\end{array}$

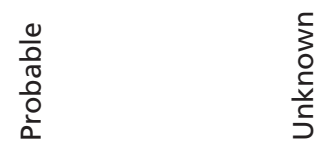

운

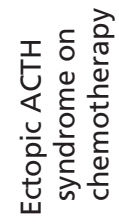

$\frac{n}{\sqrt{\sigma}}$
$\frac{0}{0}$
$\mathscr{8}$

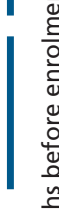

这

式

齿离

点

U

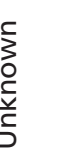

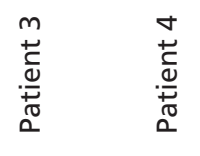

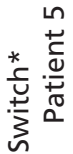

Ч্ৰ

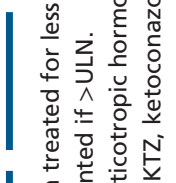

$\frac{5}{5}$

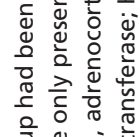

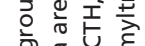

का

产

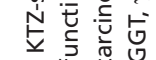

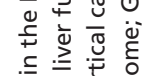

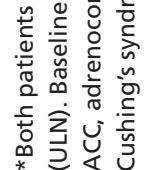




\section{Other safety variables}

Overall, 34 patients (31.5\%) experienced at least one adverse drug reaction (ADR). The majority (50) of the 79 ADRs identified were related to abnormal liver function tests. The remaining 29 ADRs were 'drug ineffective' in 13 patients, adrenal insufficiency (7 patients), joint pain/stiffness (4 patients), headache (2 patients) and erythema, alopecia, pruritus, asthenia and dyspepsia (1 patient each). Note that any individual patient may experience more than one ADR.

Thirteen patients experienced fifteen serious ADRs, including six cases of liver injury (described earlier), eight cases of adrenal insufficiency (all in the KTZnaïve cohort) and one case of headache. The cases of adrenal insufficiency may in some cases correspond to the intention of the physician to block and replace adrenal function in order to recover control of severe hypercortisolaemia rapidly. In this context, all patients had received relatively high doses of KTZ (600-1000 mg), which were reduced for only one patient, discontinued for two patients and maintained unmodified for the remaining four. Importantly, three of these patients were also treated with another steroidogenesis inhibitor.
Overall, nine deaths were reported after starting KTZ treatment, none of which was considered related to KTZ treatment. These deaths were attributed to cancer progression (three cases), pulmonary embolism (two cases) and septic shock, occlusive syndrome, tumoural syndrome and respiratory distress (one case each).

\section{Discussion}

Little information is available about the hepatic safety of KTZ in patients with CS who are potentially exposed to higher doses and for longer duration than patients using KTZ as an acute antifungal treatment. The CUP provided a framework to collect such data in a relatively large sample of patients in France. In this prospective study, we have described changes in liver function markers and their evolution over time in patients with CS treated with KTZ at daily doses ranging from 200 to $1200 \mathrm{mg}$ (median $600 \mathrm{mg}$ ) for periods of up to one year. In this population, no unanticipated hepatic safety issue was observed following treatment with KTZ. No deaths related to KTZ use occurred. Observed increases in transaminases or ALP
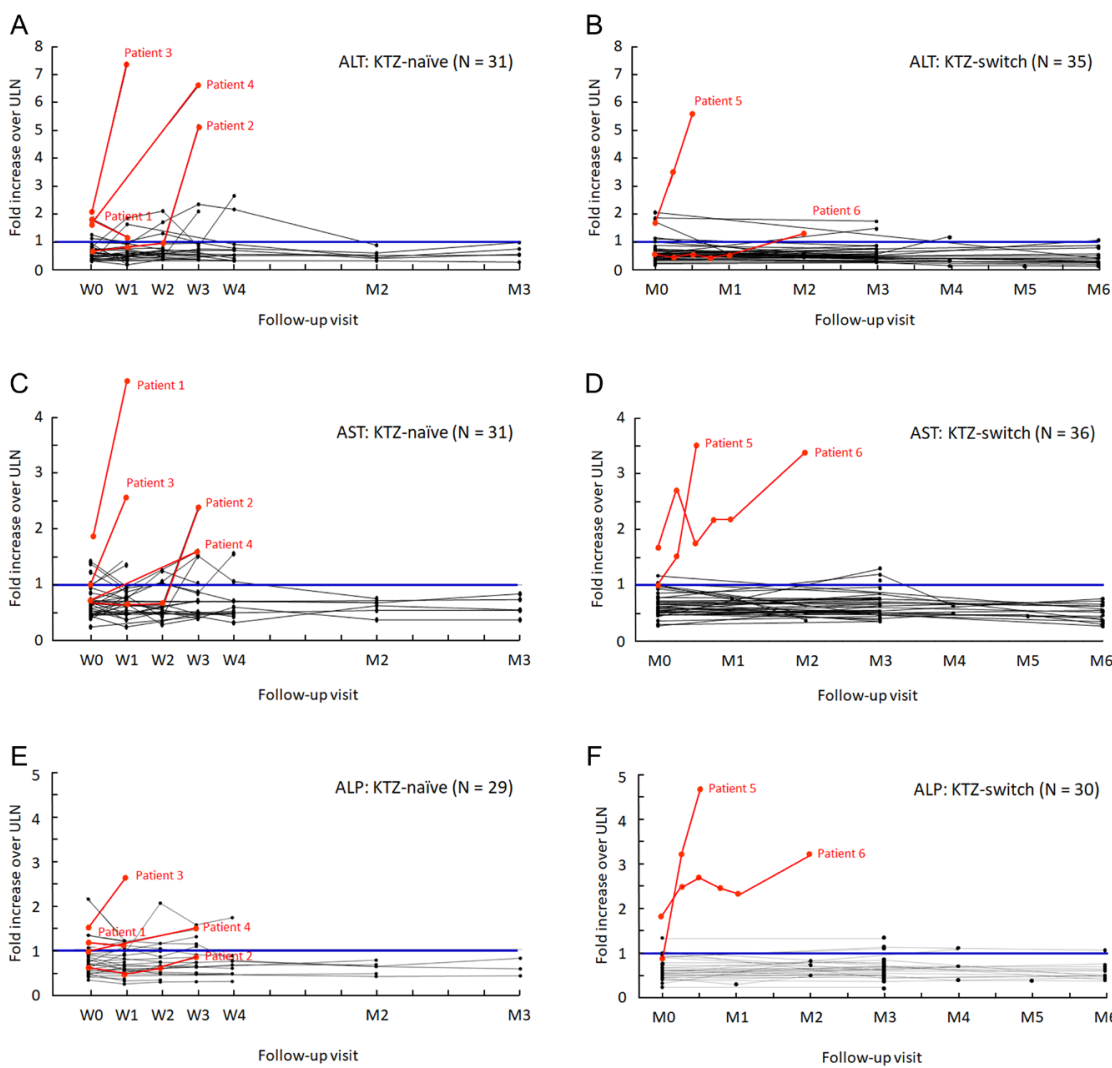

D
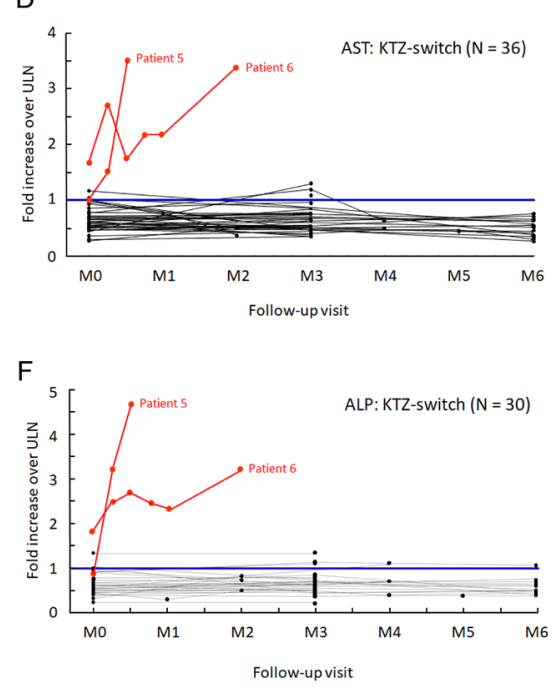

\section{Figure 2}

Evolution of transaminases and alkaline phosphatase from baseline to the last available visit in individual CS patients. Each line represents an individual patient. Only patients for whom the baseline value, at least one post-treatment value and the normal range were documented are presented. In the KTZ-switch cohort, only patients treated for over six months before inclusion in the CUP are represented (none of the patients treated for less than six months in the KTZ-switch group presented an elevation of liver enzymes > $3 \times$ ULN). M, month; W, week; W0: Initiation of KTZ-CUP treatment. The horizontal blue line represents the upper limit of normal (ULN). The red lines indicate the evolution of liver enzyme levels for the six individual patients listed in Table 4. A, C, E: naïve cohort; B, D, F: switch cohort. A, B: alanine aminotransferase; C,D: aspartate aminotransferase; E, F: alkaline phosphatase. 
were generally mild or moderate, usually occurred within the first month of treatment initiation and were in the majority of cases asymptomatic. Although the rate of treatment discontinuation was relatively high (36.1\%), the reason for discontinuation was most frequently unrelated to treatment with $\mathrm{KTZ}$, for example, the death of the patient, which accounted for around one-quarter of discontinuations or preparation for surgery for ablation of the adenoma. Eleven discontinuations $(28.2 \%$ of all discontinuations and $10.2 \%$ of treated patients) were treatment related, including four cases of inefficacy, three cases of liver injury (see below), two cases of adrenal insufficiency and two other cases due to ADRs.

Six cases of liver injury were documented in our study, four in the KTZ-naïve cohort and two in the KTZ-switch cohort. The two patients in the KTZ-switch cohort, one of whom developed jaundice, presented with a cholestatic pattern of injury, which would be inconsistent with the hepatocellular pattern typical of KTZ-associated injury (16). Both patients had abnormalities in liver function tests at baseline (13). In the patients in the KTZ-naïve cohort, liver injury occurred within around one month of KTZ initiation. This is consistent with findings from a previous study (16), in which $60 \%$ of 55 cases of KTZassociated hepatic injury appeared within the first six weeks of therapy (range: 1 week-71/2 months). Only one case, with a hepatocellular pattern of injury, was symptomatic, presenting non-specific signs indicative of hepatotoxicity (nausea and vomiting), which resolved upon discontinuation of KTZ. In the remaining three KTZ-naïve patients, there was evidence of liver metastases or pre-existing elevations in transaminases (or both), which may have contributed to the injury observed. In the patients in whom KTZ was discontinued, liver enzymes normalised. It is of note that the initial dose of KTZ was higher than the recommended starting dose of $400-600 \mathrm{mg}$ in three of these patients and pre-existing elevations of liver enzymes, in two cases exceeding $2 \times$ ULN, were present in five. A causal relationship with KTZ was questionable in certain liver injury cases due to the presence of identified liver metastases (two patients) or mild abnormalities in liver function tests (five patients) already present at inclusion. Pre-existing undiagnosed subclinical liver disease, such as steatosis or viral hepatitis, in these patients cannot be excluded. Nevertheless, these observations emphasise the importance of performing liver function tests before starting treatment and respecting carefully the prescribing recommendations provided by the manufacturer in the summary of product characteristics.
Given the low number of subjects with liver injury or elevated transaminases, it is difficult to draw firm conclusions on possible dose relationships. In previous reports, no clear dose relationship has been established for the hepatic impact of ketoconazole (11). Nonetheless, it is noteworthy that five of the six patients who developed liver injury had all been exposed to a dose of $\geq 800 \mathrm{mg}$, either as the initial dose or as a rapid up-titration. This may have been required in order to control cortisolaemia in severely ill patients. However, the prescribing information for ketoconazole specifies that the recommended starting dose is $400-600 \mathrm{mg} /$ day and that this dose can be increased to 800 $1200 \mathrm{mg} /$ day on an individual basis to ensure satisfactory control of cortisolaemia, although it is recommended that any dose increases should be made in $200 \mathrm{mg}$ steps at weekly to monthly intervals. Our findings reinforce the importance of respecting the recommended starting dose and gradual up-titration with a careful assessment of the hepatic risk at each step, on a case-by-case basis.

In our study, asymptomatic elevated levels of liver function markers appeared in around one-third of patients starting KTZ, although these elevations were mild in the majority of cases and only fulfilled the criteria for liver injury in the six patients discussed above. All cases of elevations of transaminases $\geq 2 \times$ ULN occurred within the first month of treatment in the KTZ-naïve cohort and were very rare in the patients in the KTZ-switch cohort who had been treated for over six months. Our findings can be compared with those of a retrospective cohort of 200 patients with CS treated with KTZ in France between 1995 and $2012(19,20)$, in which elevations of transaminases $<5 \times$ ULN were observed in $13.5 \%$ of patients and increases $\geq 5 \times$ ULN in $2.5 \%$. The proportion of patients with elevated transaminases was somewhat lower than that in our study, which may reflect the relative sensitivities of retrospective and prospective data collection. All these elevations were identified during the first month of treatment and all normalised after discontinuation of KTZ or dose reduction. No association between rises in transaminase levels and KTZ dose was observed. These findings in patients with CS are broadly consistent with the literature on hepatic function in patients using ketoconazole as an antifungal, in which liver enzyme elevations have also generally been observed early and are rare after six months of continuous treatment $(13,15,16,17)$.

It is difficult to compare the observed incidence of liver injury in patients with CS starting KTZ with incidence in patients using this drug as an antifungal, due to differences in case definitions and study design. A metaanalysis of data from 204 studies of use of KTZ principally 


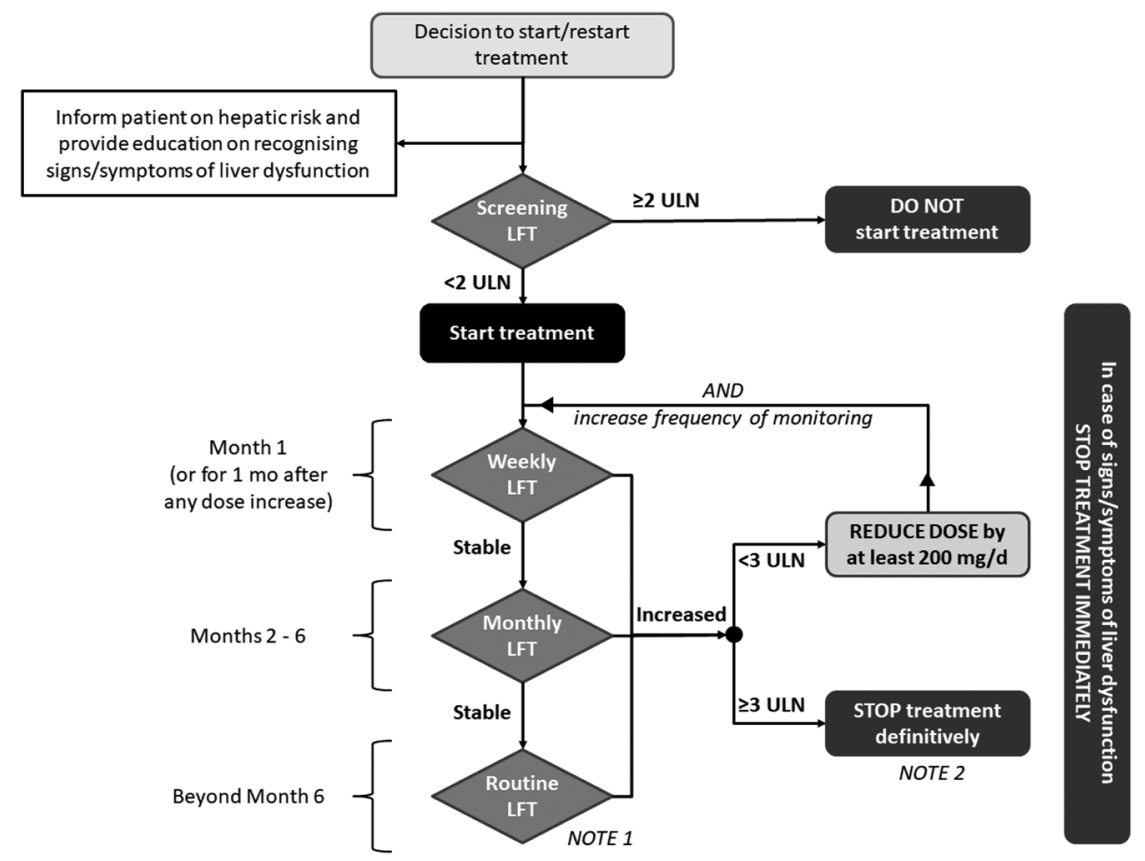

\section{Figure 3}

Algorithm for monitoring of liver function in patients with Cushing's syndrome starting treatment with ketoconazole. LFT, Liver function tests: alanine aminotransferase, aspartate aminotransferase, $\gamma$-aminotransferase, alkaline phosphatase and bilirubin; ULN, upper limit of normal. 'Depending on the patient profile and clinical status.

${ }^{2}$ Ketoconazole should not be reintroduced even after normalisation of liver function tests. as an antifungal (11) reported an incidence rate of 3.6$4.2 \%$, with no obvious dose-response relationship, but the included studies widely in terms of design. The only prospective clinical trial that has specifically evaluated liver function in 137 patients treated with KTZ as an antifungal reported that $17.5 \%$ (95\% CI: $11.1-23.9 \%)$ of patients presented asymptomatic elevations of ALT > ULT and a further $2.9 \%$ (95\% CI: 0.1-5.7\%) showed elevations with clinical symptoms (21). In this study, the daily dose was $200 \mathrm{mg}$ and the mean time to onset of elevated ALT was 6.4 weeks. A baseline transaminase level $<\mathrm{ULN}$ was an entry criterion for this study.

Our findings emphasise the need for clinical and biochemical monitoring at regular intervals for evidence of hepatic injury or of mild asymptomatic increases in liver enzymes, which are by far the most frequent picture observed in patients treated with KTZ. Weekly monitoring should be performed at least over the first month and then on a monthly basis for up to six months, as recommended in the prescribing information. An algorithm illustrating the recommended monitoring of liver function in these patients is provided in Fig. 3. Patients with liver enzyme levels between ULN and $2 \times$ ULN at baseline need particularly close follow-up. The appearance of any signs or symptoms suggestive of liver injury, such as unusual fatigue, abdominal pain, nausea, vomiting or jaundice, should prompt immediate monitoring of liver enzymes. In case of dose escalation, close monitoring of liver enzymes should be re-established, since this has been reported to trigger elevations of transaminases in patients who have previously tolerated KTZ therapy well with respect to hepatic function $(13,17,19)$. We believe that KTZ should be discontinued immediately in patients whose transaminases, ALP or bilirubin increase above $3 \times \mathrm{ULN}$ in order to avoid severe liver injury. In patients with increases that did not reach $3 \times \mathrm{ULN}$, the dose should be reduced by at least $200 \mathrm{mg}$. In the literature $(13,14,15$, $16,17)$, fatal cases have been reported particularly when KTZ is continued despite marked liver enzyme elevation.

It should be noted that CS also carries a risk of liver injury independently of the use of KTZ (22) and is not uncommonly associated with non-alcoholic steatohepatitis, which may evolve into fibrosis or cirrhosis $(23,24)$. Whether long-term treatment with KTZ aggravates this risk is unknown. This cannot easily be addressed in studies such as ours since progression of structural liver disease may not be associated with dramatic changes in transaminases and may require a longer timeframe than that of the present study. Physicians should be aware of the risk of structural liver disease in patients with longstanding CS and consider episodic assessment with non-invasive techniques such as sonography or transient elastography (25), or in case of suggestive clinical manifestations. Assessment of potential subclinical liver disease could be useful prior to initiating treatment with ketoconazole, notably in patients with more severe and longstanding CS.

The CUP was established to give patients with a rare disease the opportunity to be treated and to describe the safety of KTZ in everyday care in France. 
The strengths and limitations of the study reflect this context. Among the strengths, the study enrolled a relatively large number of patients considering the rarity of the condition (26). The study was performed in a naturalistic treatment setting reflecting 'real-life' use of KTZ in an otherwise unselected sample of patients with a confirmed diagnosis of CS without contraindication to treatment with KTZ and treated with a wide range of doses. The data were collected prospectively and changes in liver function tests were analysed on an individual patient basis. The clinical and biochemical findings were evaluated by an experienced hepatologist. The principal limitation was the relatively high amount of missing data especially in the KTZ-switch cohort. The CUP was not a formal clinical trial with established visits and formal monitoring and, although participating physicians were asked to provide follow-up data to the coordinating centre, this could not be enforced. As a result, less data than expected were available for analysis. It should be borne in mind that physicians had been prescribing KTZ to patients with CS on an offlabel basis for over thirty years without any obligation to provide follow-up data. Another limitation was that the liver function tests were not centralised and were conducted according to the standard practice of the 100 participating centres, which may have led to some imprecision in the consolidation of the data. Finally, it was not possible to take into account other risk factors for abnormal liver function tests, such as alcohol intake or concomitant treatment with other hepatotoxic drugs, which may also be present in these patients.

Although KTZ has been used off-label for the treatment of CS for several decades, it is now authorised in the EU for the treatment of CS in patients over the age of 12 years. These new data collected prospectively highlight the need for close monitoring of liver enzymes especially at the beginning of treatment and up to six months. If care is taken to monitor liver function effectively, KTZ can be used safely in patients with CS.

Supplementary data

This is linked to the online version of the paper at https://doi.org/10.1530/ EJE-17-0886.

\section{Declaration of interest}

$S \mathrm{~S}$ is an employee of HRA Pharma and RC was an employee of this company at the time of the study. J B, F C, O C, M C V and J Y have received reimbursement from HRA Pharma for travel in connection with participation in Steering Committee meetings for this study. O C and $\mathrm{F}$ C have participated as remunerated speakers at conference symposia sponsored by HRA Pharma. F C has received consultancy fees from HRA Pharma. J B has participated in Advisory Boards for Atterocor and Shire and on speaker panels for Novartis and HRA Pharma. J B and F C have received research grants from Novartis.

\section{Funding}

This work was supported by HRA Pharma.

\section{Author contribution statement}

The academic authors ( $\mathrm{Y}, \mathrm{J} B, \mathrm{O}$ C, M C V, F C) constituted the scientific advisory panel for this study and recruited patients into the programme. R C, an employee of the study sponsor at the time of the study, oversaw the design of the protocol and the conduct of the study. S S oversaw the final data analysis and the preparation of this manuscript. All authors contributed to the preparation of the manuscript, reviewed and approved the final version.

\section{Acknowledgements}

The authors wish to express their gratitude to all the patients and clinic staff who have participated in this cohort study. The authors are grateful to all the physicians who entered patients into this Compassionate Use Programme: J Abeillon (Bron), C Ajzenberg (Créteil), J-M Andrieu (Béziers), A-S Arbey (Lons-le-Saunier), F Archambeaud (Limoges), G Arnault (Vannes), J Bacchetta (Bron), E Baechler-Sadoul (Nice), F Bakiri (St Denis de la Réunion), M Batisse-Lignier (Clermont-Ferrand), C Baudry (Bobigny), E Benamo (Avignon), A Bennet (Toulouse), A Berdelou (Villejuif), J Bertherat (Paris), A Boehna (Mulhouse), F Borson-Chazot (Bron), N Bourcigaux (Paris), C Bourquard (Le Puy-en-Velay), L Bouys (Paris), C Bremont-Weill (Paris), L Bricaire (Paris), T Brue (Marseille), D Buliga (St Malo), P Cabaret (Lomme), P Caron (Toulouse), M CerroMartinez (St Jean de Verges), C Chambre (Bobigny), M Chardonnet (St Pierre de la Réunion), J Chatelin (Nancy), C Clavel (Toulouse), C Coffin (Périgueux), R Cohen (St Denis), C Collet-Gaudillat (Le Chesnay), C Cortet (Lille), A-L Coulon (Grenoble), F De Boisvilliers (Montpellier), A DeckerBellaton (Gleizé), B Delemer (Reims), M De Menthon (Paris), V Degros (Valenciennes), T Deneuville (Paris), R Desailloud (Amiens), G Di Pietro (Monaco), C Do Cao (Lille), B Donadille (Paris), M Dolz (Brest), P DubrayLongeras (Clermont-Ferrand), E Dutertre (Montpellier), H Du Rostu (Rézé), J El Farkh (Aubenas), G Faure (Niort), P Finichel (Nice), N Gaits (Tarbes), F Galland (Rennes), S Genc (Paris), E Ghanassia (Sète), J-J Girard (Loches), G Gravis (Marseille), L Groza (Metz), F Grunenberger (Strasbourg), C Guiheneuf (Bois-Guillaume), L Guignat (Paris), M Guigui (Trévenans), A Guilhem (Montpellier), M Joubert (Caen), C Jublanc (Paris), P Kamenicky (Kremlin-Bicêtre), V Kerlan (Brest), Y Khalfallah (St Etienne), G Lambrey (Beauvais), E Landau (Nantes), C Lautridou (Paris), H Lefebvre (Rouen), M Le Bras (Nantes), A-C Le Guillou (Lille), C Le Pommelet (Cayenne), F Léonard (Arras), L Lin (La Trinité, Martinique), F Luca (Strasbourg), C Lunogo (Villejuif), A Maisin (Paris), J-C Maiza (St Pierre de la Réunion), M Marty (Pessac), P Meliani (Mayotte), S Menon (Rouen), B Mestre (PierreBénite), B Mignot (Besançon), S Millot (Mulhouse), I Morange (Marseille), M Moret (Bron), N Morlet-Barla (Marseille), H Narbonne (Marseille), B Néraud (Suresnes), N Néri (Nanterre), P Niccoli (Marseille), M L Nunes (Pessac), S Oudard (Paris), S Papadopoulou (Bobigny), J-M Petit (Dijon), G Petit-Aubert (Le Chesnay), A Perrin (Roanne), M Philippon (Marseille), C Pillegrand (Bondy), L Potton (Grenoble), G Raverot (Bron), M L Rodes (Niort), Y Reznik (Caen), J L Sadoul (Nice), S Salenave (Kremlin-Bicêtre), M Saraval-Gross (Amiens), J Sarfati (Kremlin-Bicêtre), J Savel (Pessac), M A Schletzer (Laval), S Schneebeli (St Pierre de la Réunion), F Schillo (Besançon), A Smagala (Colmar), E Sonnet (Brest), R Teissier (Brest), M P Tessier (Limoges), F Trulli (Villejuif), C Vackrine (Montbrison), D Vezzosi (Toulouse), A Viard (Reims), A Villeneuve (Orléans), G Weryha (Nancy), M Zalzali (Reims). 


\section{References}

1 Lacroix A, Feelders RA, Stratakis CA \& Nieman LK. Cushing's syndrome. Lancet 2015386 913-927. (https://doi.org/10.1016/ S0140-6736(14)61375-1)

2 Pivonello R, Isidori AM, De Martino MC, Newell-Price J, Biller BM \& Colao A. Complications of Cushing's syndrome: state of the art. Lancet Diabetes and Endocrinology 20164 611-629. (https://doi. org/10.1016/S2213-8587(16)00086-3)

3 Pivonello R, De Leo M, Cozzolino A \& Colao A. The treatment of Cushing's disease. Endocrine Reviews 201536 385-486. (https://doi. org/10.1210/er.2013-1048)

4 Fleseriu M \& Petersenn S. Medical therapy for Cushing's disease: adrenal steroidogenesis inhibitors and glucocorticoid receptor blockers. Pituitary 201518 245-252. (https://doi.org/10.1007/s11102014-0627-0)

5 Lo Re V 3rd, Carbonari DM, Lewis JD, Forde KA, Goldberg DS, Reddy KR, Haynes K, Roy JA, Sha D, Marks AR et al. Oral azole antifungal medications and risk of acute liver injury, overall and by chronic liver disease status. American Journal of Medicine 2016129 283.e285-291.e285. (https://doi.org/10.1016/j. amjmed.2015.10.029)

6 Pont A, Williams PL, Loose DS, Feldman D, Reitz RE, Bochra C \& Stevens DA. Ketoconazole blocks adrenal steroid synthesis. Annals of Internal Medicine 198297 370-372. (https://doi.org/10.7326/00034819-97-3-370)

7 Angeli A \& Frairia R. Ketoconazole therapy in Cushing's disease. Lancet 19851 821. (https://doi.org/10.1016/S0140-6736(85)91482-5) 8 Bradbrook ID, Gillies HC, Morrison PJ, Robinson J, Rogers HJ \& Spector RG. Effects of single and multiple doses of ketoconazole on adrenal function in normal subjects. British Journal of Clinical Pharmacology 198520 163-165. (https://doi. org/10.1111/j.1365-2125.1985.tb05051.x)

9 Dandona P, Mohiuddin J \& Prentice HG. Ketoconazole and adrenocortical secretion. Lancet 19851 227. (https://doi.org/10.1016/ S0140-6736(85)92073-2)

10 Couch RM, Muller J, Perry YS \& Winter JS. Kinetic analysis of inhibition of human adrenal steroidogenesis by ketoconazole. Journal of Clinical Endocrinology and Metabolism 198765 551-554. (https:// doi.org/10.1210/jcem-65-3-551)

11 Yan JY, Nie XL, Tao QM, Zhan SY \& Zhang YD. Ketoconazole associated hepatotoxicity: a systematic review and meta-analysis. Biomedical and Environmental Sciences 201326 605-610. (https://doi. org/10.3967/0895-3988.2013.07.013)

12 Greenblatt HK \& Greenblatt DJ. Liver injury associated with ketoconazole: review of the published evidence. Journal of Clinical Pharmacology 201454 1321-1329. (https://doi.org/10.1002/jcph.400)

13 Lewis JH, Zimmerman HJ, Benson GD \& Ishak KG. Hepatic injury associated with ketoconazole therapy. Analysis of 33 cases. Gastroenterology 198486 503-513.

14 Garcia Rodriguez LA, Duque A, Castellsague J, Perez-Gutthann S \& Stricker BH. A cohort study on the risk of acute liver injury among users of ketoconazole and other antifungal drugs. British Journal of
Clinical Pharmacology 199948 847-852. (https://doi.org/10.1046/ j.1365-2125.1999.00095.x)

15 Janssen PA \& Symoens JE. Hepatic reactions during ketoconazole treatment. American Journal of Medicine 198374 80-85. (https://doi. org/10.1016/0002-9343(83)90519-3)

16 Stricker BH, Blok AP, Bronkhorst FB, Van Parys GE \& Desmet VJ. Ketoconazole-associated hepatic injury. A clinicopathological study of 55 cases. Journal of Hepatology 19863 399-406. (https://doi. org/10.1016/S0168-8278(86)80495-0)

17 Lake-Bakaar G, Scheuer PJ \& Sherlock S. Hepatic reactions associated with ketoconazole in the United Kingdom. BMJ 1987294 419-422. (https://doi.org/10.1136/bmj.294.6569.419)

18 European Medicines Agency. European Medicines Agency recommends suspension of marketing authorisations for oral ketoconazole. Benefit of oral ketoconazole does not outweigh risk of liver injury in fungal infections. EMA/458028/2013. 2013. (https://www.ema.europa.eu/docs/en_GB/document_library/Press_ release/2013/07/WC500146613.pdf)

19 Castinetti F, Guignat L, Giraud P, Muller M, Kamenicky P, Drui D, Caron P, Luca F, Donadille B, Vantyghem MC et al. Ketoconazole in Cushing's disease: is it worth a try? Journal of Clinical Endocrinology and Metabolism 201499 1623-1630. (https://doi.org/10.1210/ jc.2013-3628)

20 Castinetti F, Morange I, Jaquet P, Conte-Devolx B \& Brue T. Ketoconazole revisited: a preoperative or postoperative treatment in Cushing's disease. European Journal of Endocrinology 2008158 91-99. (https://doi.org/10.1530/EJE-07-0514)

21 Chien RN, Yang LJ, Lin PY \& Liaw YF. Hepatic injury during ketoconazole therapy in patients with onychomycosis: a controlled cohort study. Hepatology 199725 103-107. (https://doi.org/10.1002/ hep.510250119)

22 Kamenicky P, Droumaguet C, Salenave S, Blanchard A, Jublanc C, Gautier JF, Brailly-Tabard S, Leboulleux S, Schlumberger M, Baudin E et al. Mitotane, metyrapone, and ketoconazole combination therapy as an alternative to rescue adrenalectomy for severe ACTH-dependent Cushing's syndrome. Journal of Clinical Endocrinology and Metabolism 201196 2796-2804. (https://doi. org/10.1210/jc.2011-0536)

23 Rockall AG, Sohaib SA, Evans D, Kaltsas G, Isidori AM, Monson JP, Besser GM, Grossman AB \& Reznek RH. Hepatic steatosis in Cushing's syndrome: a radiological assessment using computed tomography. European Journal of Endocrinology 2003149 543-548. (https://doi.org/10.1530/eje.0.1490543)

24 Hazlehurst JM \& Tomlinson JW. Non-alcoholic fatty liver disease in common endocrine disorders. European Journal of Endocrinology 2013 169 R27-R37. (https://doi.org/10.1530/EJE-13-0296)

25 Kaswala DH, Lai M \& Afdhal NH. Fibrosis assessment in Nonalcoholic Fatty Liver Disease (NAFLD) in 2016. Digestive Diseases and Sciences 201661 1356-1364. (https://doi.org/10.1007/s10620016-4079-4)

26 Steffensen C, Bak AM, Rubeck KZ \& Jorgensen JO. Epidemiology of Cushing's syndrome. Neuroendocrinology 201092 (Supplement 1) 1-5. (https://doi.org/10.1159/000314297)

Received 25 October 2017

Revised version received 14 February 2018

Accepted 22 February 2018 